\title{
Body proportions as possible predictors for free gracilis one-stage facial reanimation
}

\author{
Bohac $\mathrm{M}^{1}$, Palkovic $\mathrm{M}^{2}$, Fedeles $\mathrm{J}^{1}$, Hodosy $\mathrm{J}^{3,4}$ \\ Clinics of plastic and reconstructive surgery, Faculty of Medicine, Comenius University and University \\ Hospital Bratislava, Bratislava, Slovakia. hodosy@gmail.com
}

\begin{abstract}
BACKGROUND: Gracilis muscle and its motor nerve belongs to most commonly used flap for facial reanimation. However, it is performed in two steps, which is time consuming. One stage technique can be also performed, but the length of the motor nerve cannot be currently determined before surgery.

AIM: The present study was conducted in order to evaluate the body composition on the length and suitability of the motor nerve of gracilis muscle for one stage facial reanimation.

METHODS: The gracilis flaps along with the motoric nerve were dissected from 20 fresh cadavers (6 females, 14 males). The length of the lower extremity from superior iliac anterior spine to the bottom of the heel and BMI were measured. Regression analysis of lower extremity length and BMI to the actual length of the motor nerve of gracilis flap was performed.

RESULTS: The linear regression analysis showed a positive correlation between the length of the lower limb and the size of the motor nerve length $(r=0.5060, p<0.05)$, as well as between the BMI and the size of the motor nerve length $(r=0.5073, p<0.05)$. Also, the males had longer motor nerve when compared to females by $13 \%(p<0.05)$. No difference between females and males in BMI was observed.

CONCLUSION: The length from the superior iliac anterior spine, BMI and gender seemed to be potential factors that could help to predict the length of the gracilis flap motor nerve for the one stage facial reanimation. However, further studies evaluating other anatomical factors and validating the possible prediction rule for one stage reanimation success are needed (Fig. 3, Ref. 14). Text in PDF www.elis.sk. KEY WORDS: gracilis flap, motor nerve length, one stage facial reanimation.
\end{abstract}

\section{Introduction}

Long standing facial paralysis represents apart from functional, also aesthetic and psychological problem for the patients (1). Although wide range of causes and clinical appearance advocates individualized approach to the treatment, generally, facial nerve paralysis can be divided into either congenital or acquired (2). The treatment modalities include the static and dynamic options, the latter one to be the treatment of choice whenever possible, since they truly reanimate the face. The gracilis free-muscle transfer with cross-facial nerve grafting represents the gold standard. This is usually performed in two steps, the first being the nerve trans-

${ }^{1}$ Clinics of plastic and reconstructive surgery, Faculty of Medicine, Comenius University and University Hospital Bratislava, Bratislava, Slovakia, ${ }^{2}$ Institute of pathology, Faculty of Medicine, Comenius University, Bratislava, Bratislava, Slovakia, ${ }^{3}$ Institute of molecular biomedicine, Faculty of Medicine, Comenius University, Bratislava, Slovakia, and ${ }^{4}$ Emergency department Ruzinov, University Hospital Bratislava, Bratislava, Slovakia

Address for correspondence: J. Hodosy, MD, MSc, PhD, MPH, Institute of molecular biomedicine, Medical Faculty, Comenius University, Sasinkova 4, SK-813 72 Bratislava, Slovakia.

Phone: +421.2.59357296

Acknowledgements: The project was supported by the grant No: ITMS 26240220087. fer and the second being the muscle flap transplantation (3). The time between these two steps is 6-24 months, and represents a major disadvantage of a two-stage facial reanimation. Therefore, a one-stage method was developed by O’Brien and Kumar (4), leaving the two-stage method as a choice of treatment in young and thinner children (5).

Nevertheless, the one stage method of facial reanimation has a major flaw back in the length of the motor nerve, which cannot be predicted before surgery. Additionally, the gracilis muscle often has more than one neurovascular pedicle, which might impede the harvest of the gracilis flap. Indeed, other muscle flaps had been used to overcome this problem, including latissimus dorsi muscle (6) or free abductor hallucis muscle (7). However, gracilis muscle with its motor nerve still remains the most appropriate flap possibility, despite a lack of predicting tools.

Body composition and anthropometrics can serve as an interesting and easy way to obtain parameters. For example, body mass index as a marker of obesity, which is yet another risk factor for stroke (8), myocardial infarction (9) and many others are widely used. On the other hand, the choice for gracilis flap as the first choice still remains solely upon the experience of the plastic surgeon rather on critical review and planning of the surgery itself. Preoperative prediction of motor nerve length of free gracilis flap is key to determine the potential outcome and 


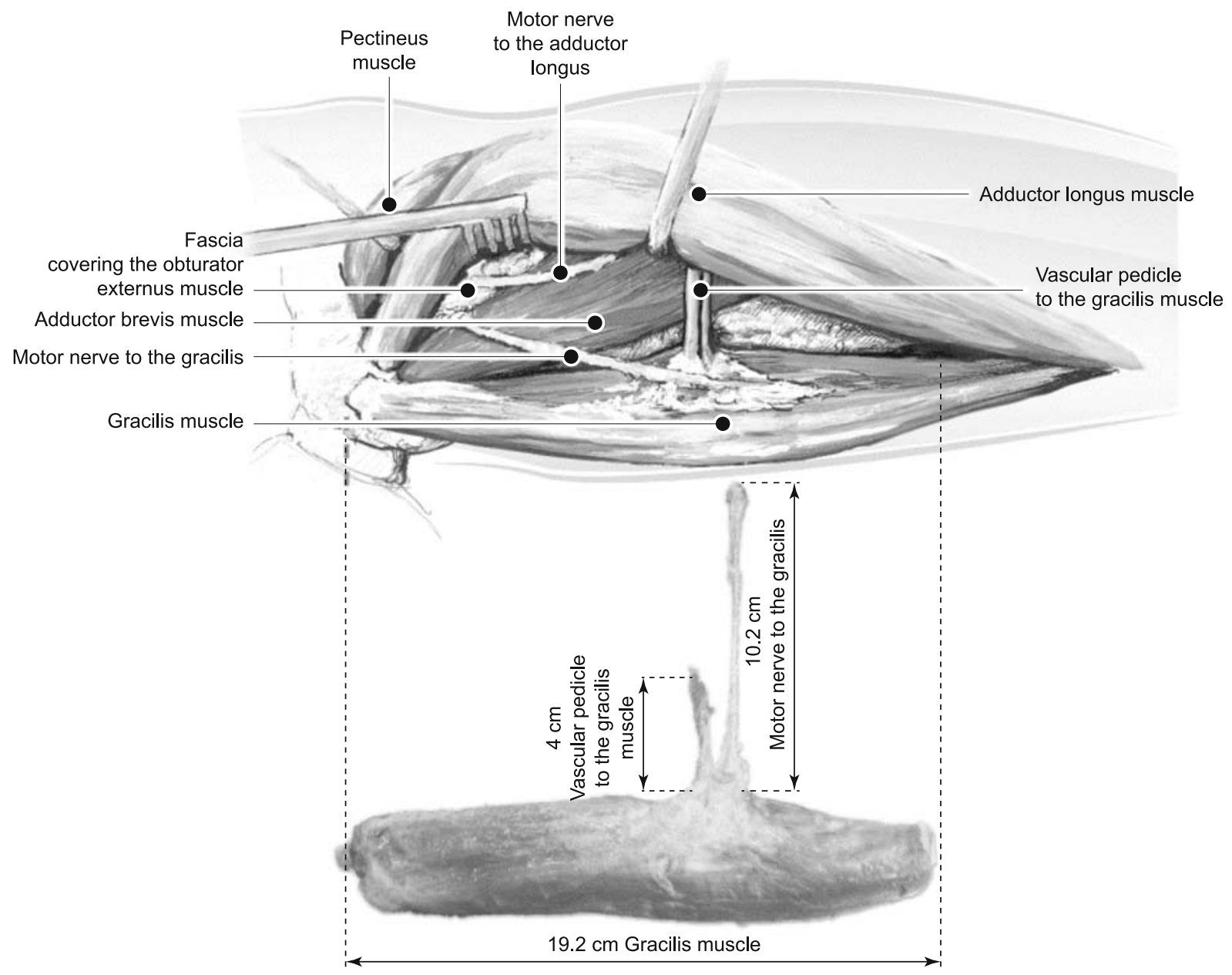

Fig. 1. Schematics of first pedicle of harvesting the gracilis muscle flap and its first pedicle.

plan the surgery technique. Various body lengths are started to be used in, for example, graft selection for anterior cruciate ligament repair (10) or prediction of height from other body parts measurements (11).

Therefore, the aim of this wor kwas to report the possible predictive measures and to standardize the location of the first and most commonly used neurovascular pedicle of the gracilis flap for the use in one-stage facial reanimation.

\section{Subjects and methods}

Twenty left lower-limbs were dissected from 20 fresh cadavers, six females and fourteen males at Institute of pathological anatomy of the Faculty of Medicine, Comenius University in Bratislava. The dissections were performed as follows: A simple incision was made along the medial side of the thigh up to the inguinal fold. The skin and fascia of the medial side of the thigh were retracted, allowing the exposure of the adductor muscles of the thigh. The neurovascular pedicle for the gracilis was exposed after retracting the adductor longus, and the motor nerve for the gracilis was dissected proximally at the obturator foramen. For each cadaver, the length of the motor nerve from the gracilis neurovascular hilum to the obturator foramen and the length of the lower extremity were measured from superior iliac anterior spine (SIAS) down to the bottom of the heel. Facial measurement included distance between the lateral orbital rims, where after vertical line projection the branches of facial nerve are sufficiently large. The height and weight were taken from the history documents of the cadavers, and subsequently the BMI was calculated.

\section{Statistical analysis}

GraphadPrism 6.0 (La Jolla, CA, USA) was used for figures and statistical analysis. The data were analyzed using linear regression and Spearman correlation coefficients were calculated. Mann-Whitney two-sided test was used for assessment of continuous variables between females and males. $\mathrm{p}<0.05$ was considered as statistically significant. Where appropriate, data are presented as median \pm interquartile range (IQR). 
(A)

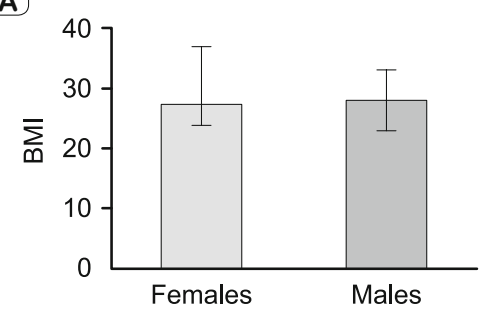

(B)

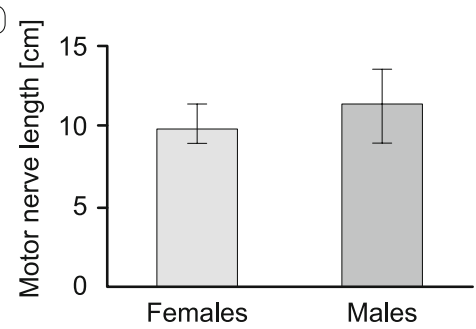

(C)

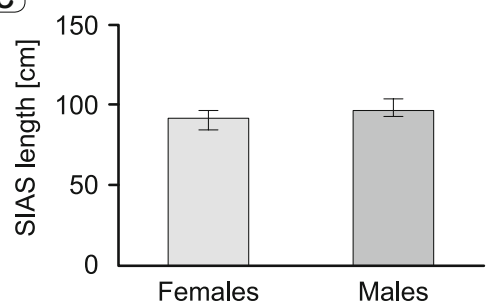

Fig. 2. The difference between females and males in A) BMI and B) length of the motor nerve. * denotes $\mathbf{p}<0.05$, data are presented as median \pm IQR.
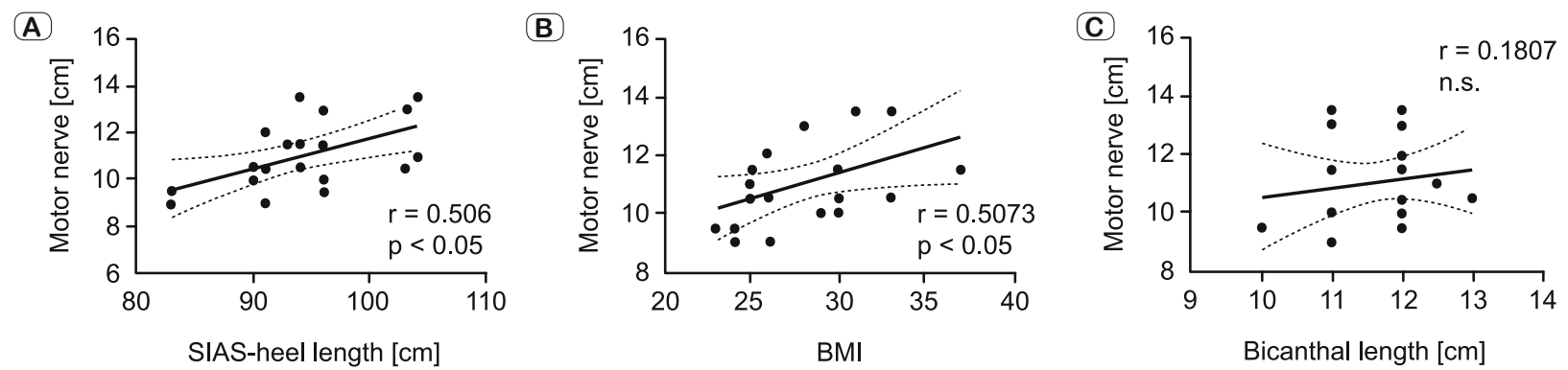

Fig. 3. The correlation between motor nerve length and A) lower extremity length, B) BMI, C) bicanthal length. $r$ - correlation coefficient, n.s. - not significant.

\section{Results}

Figure 1 represents a schematics showing how the distance between the first pedicle and obturator foramen was measured. No significant difference was observed in BMI between females and males (Fig. 2A). The median for BMI in females was 27.5 (IQR: 25.50; 31.75) and males 28 (IQR: 25.00; 30.25). On the other hand, the length of the motor nerve was longer by $13 \%$ in males when compared to females ( $\mathrm{p}<0.05$ ) (Fig. 2B). The median for the motor nerve length in females was 10.0 (IQR: 9.37; 10.75) and 11.5 (IQR: 10.50; 13.00). No significant difference (Fig. 2C) was found in the length of the lower limb between females (median 90.50, IQR: 88.25; 96.00) and males (median 95.00, IQR: 92.50; 103). The motor nerve length was positively correlated with the lower extremity length from SIAS to the bottom of the heel (Fig. 3A) $(r=0.5060, p<0.05)$ and also with the BMI (Fig. 3B) $(r=$ $0.5073, p<0.05)$. However, the length of the motor nerve was not associated with the bicanthal length (Fig. 3C) ( $r=0.1807$, n.s.).

\section{Discussion}

The dynamic correction of the facial paralysis includes achievement of maximal symmetry and spontaneous smile. A graft from the same host, i.e. autografting, is the optimal way for facial paralysis treatment, since the preserved architecture of the nerve, which further serves as a skeleton for neuroregeneration. Clinical descriptions of motor to sensory nerve preference are lacking (12), however, two-stage motor nerve with gracilis flap remains the standard and most widely used technique for treat- ment of facial palsy (13). Later, several authors successfully tried to achieve the same result with one-stage procedure, shortening thus time to achieve smile and minimizing the potential negative effects of donor site surgery. Indeed, several improvements to the one-stage procedures had been invented to overcome the problem of short motor nerve of the gracilis flap. Even though, the obturator nerve still might have insufficient length, negating thus advantages of one-stage procedure (14).

To our knowledge, this is the first study that evaluated the potential predictors of the gracilis flap motor nerve using physical cues of the individual patient. Presently, the SIAS length (length of the lower extremity) and BMI had the highest prediction value for the motor nerve of gracilis flap, from the obturator foramen to gracilis muscle. Indeed, one of the study limitation is the fact, that the determination coefficients were low for both factors (R2 $=0.25$ ). Nevertheless, gender itself can lead to longer motor nerve length, males achieving longer distances. Certainly, one could argue that males being taller with longer SIAS length would have also longer motor nerve length. However, in our study, there was no difference in the length of the SIAS between genders. This suggests a gender being another independent factor for motor nerve length to take into consideration.

Taken together, our study points that SIAS length, gender and BMI could be possible prediction factors for success in onestage facial reanimation using motor nerve with gracilis muscle flap. Nevertheless, other, especially validation studies should be performed in order to confirm our findings. Also, other physical predictor cues should be taken into consideration in further studies. 


\section{$547-550$}

\section{References}

1. Lindsay RW, Bhama P, Hadlock TA. Quality-of-life improvement after free gracilis muscle transfer for smile restoration in patients with facial paralysis. JAMA Fac Plast Surg 2014; 16 (6): 419-424.

2. Westin LM, Zuker R. A new classification system for facial paralysis in the clinical setting. J Craniofac Surg 2003; 14 (5): 672-679.

3. Rab M, Haslik W, Grunbeck M et al. Free functional muscle transplantation for facial reanimation: experimental comparison between the one- and two-stage approach. J Plast Reconstr Aesth Surg 2006; 59 (8): 797-806.

4. Kumar PA. Cross-face reanimation of the paralysed face, with a single stage microneurovascular gracilis transfer without nerve graft: a preliminary report. Brit J Plast Surg 1995; 48 (2): 83-88.

5. Garcia RM, Hadlock TA, Klebuc MJ, Simpson RL, Zenn MR, Marcus JR. Contemporary solutions for the treatment of facial nerve paralysis. Plast Reconstr Surg 2015; 135 (6): 1025e-1046e.

6. Takushima A, Harii K, Asato H, Kurita M, Shiraishi T. Fifteenyear survey of one-stage latissimus dorsi muscle transfer for treatment of longstanding facial paralysis. J Plast Reconstr Aesth Surg 2013; 66 (1): 29-36.

7. Liu AT, Lin Q, Jiang $\mathbf{H}$ et al. Facial reanimation by one-stage microneurovascular free abductor hallucis muscle transplantation: personal experience and long-term outcomes. Plast Reconstr Surg 2012; 130 (2): 325-335.
8. Serena J, Segura T, Roquer J, Garcia-Gil M, Castillo J, Study A. The ARTICO study: identification of patients at high risk of vascular recurrence after a first non-cardioembolic stroke. BMC Neurol 2015; 15-28.

9. Borgeraas H, Hertel JK, Svingen GF et al. Association of body mass index with risk of acute myocardial infarction and mortality in Norwegian male and female patients with suspected stable angina pectoris: a prospective cohort study. BMC cardiovascular disorders 2014; 1468.

10. Park YB, Song YS, Kim SC, Park YG, Ha CW. The size of tibial footprint of anterior cruciate ligament and association with physical characteristics in Asian females. Arch Orthop Trauma Surg 2015;

11. De Mendonca MC. Estimation of height from the length of long bones in a Portuguese adult population. Amer J Phys Anthropol 2000; 112 (1): 39-48.

12. Iorio ML, Felder JM, 3rd, Ducic I. Anterior branch of the obturator nerve: a novel motor autograft for complex peripheral nerve reconstruction. Ann Plast Surg 2011; 67 (3): 260-262.

13. Rozen S, Rodriguez-Lorenzo A, Audolfsson T, Wong C, Cheng A. Obturator nerve anatomy and relevance to one-stage facial reanimation: limitations of a retroperitoneal approach. Plast Reconstr Surg 2013; 131 (5): 1057-1064.

14. Rodriguez Lorenzo A, Morley S, Payne AP, Tollan CJ, Soutar DS. Anatomy of the motor nerve to the gracilis muscle and its implications in a one-stage microneurovascular gracilis transfer for facial reanimation. $\mathrm{J}$ Plast Reconstr Aesth Surg 2010; 63 (1): 54-58.

Received April 13, 2016. Accepted May 6, 2016. 